\title{
INTEGRAL and RXTE view of Her X-1: towards resolving of the system's puzzles
}

\author{
D. Klochkov ${ }^{* a}$, R. Staubert ${ }^{a}$, K. Postnov ${ }^{b}$, N. Shakura ${ }^{b}$, A. Santangelo ${ }^{a}$, J. Wilms ${ }^{c}$, \\ and R. Rothschild ${ }^{d}$ \\ ${ }^{a}$ Institut für Astronomie und Astrophysik, Universität Tübingen (IAAT) \\ ${ }^{b}$ Sternberg Astronomical Institute, Moscow University \\ ${ }^{c}$ Dr. Karl Remeis-Sternwarte, Astronomisches Institut, Universität Erlangen-Nürnberg \\ ${ }^{d}$ Center for Astrophysics and Space Sciences, University of California \\ E-mail: klochkov@astro.uni-tuebingen.de
}

\begin{abstract}
Her X-1 is one of the most intensively studied accreting X-ray pulsars. The source was regularly observed with Rossi XTE since its launch in 1996 which allowed us to monitor X-ray spectral and timing properties of the source. In July-August 2005 and September 2007 Her X-1 was observed with INTEGRAL. In both cases the observations covered a substantial part of the source's MainOn states. Both satellites contributed significantly to the understanding of the physical processes in the binary. We discuss the results obtained with RXTE and INTEGRAL in the context of our current understanding of the system's phenomenology.
\end{abstract}

7th INTEGRAL Workshop

September 8-11 2008

Copenhagen, Denmark

\footnotetext{
* Speaker.
} 


\section{Introduction}

Discovered in 1972 by the Uhuru satellite (Giacconi et al. 1973; Tananbaum et al. 1972), Her X-1 is one of the most intensively studied accreting pulsars. The basic phenomenological picture of Her X-1 was established soon after its discovery: a close binary system consisting of an accreting magnetized neutron star with a $1.24 \mathrm{~s}$ spin period and a stellar companion HZ Her (first suggested by Liller 1972) - a main sequence star of the spectral type A/F (Crampton 1974). The orbital period of the system is $\sim 1.7$ days.

Due to the high orbital inclination of the system (which is seen almost edge-on) the counterorbitally precessing tilted and warped accretion disk around the neutron star covers the central $\mathrm{X}$-ray source from the observer during a substantial part of its $35 \mathrm{~d}$ precessional period. This gives rise to the periodic alternation of so-called on (high X-ray flux) and off (low X-ray flux) states. One $35 \mathrm{~d}$ (precessional) cycle contains two on states - the main-on and the short-on - separated by $\sim 7-8 \mathrm{~d}$ off state. The X-ray flux in the middle of the main-on state is $\sim 4-5$ times higher than that in the middle of the short-on. The sharp transition from the off-state to the main-on is called the turn-on of the source. Turn-ons are usually used for counting the cycles. The $35 \mathrm{~d}$ period manifests itself also in variations of the shape of X-ray pulse profiles (Soong et al. 1990b; Trümper et al. 1986; Deeter et al. 1998; Scott et al. 2000; Staubert et al. 2009a) and modulation of optical light curves (Gerend \& Boynton 1976; Howarth \& Wilson 1983).

The X-ray spectrum of Her X-1 is characterized by a power law continuum with exponential cut-off and an apparent line-like feature, which was discovered in 1976 (Trümper et al. 1978). This line is now generally accepted as an absorption feature due to resonant scattering of photons off electrons on quantized energy levels (Landau levels) in the Teragauss magnetic field at the polar cap of the neutron star. The feature is therefore often referred to as a cyclotron line or cyclotron resonant scattering feature (CRSF). Being a common feature of accreting pulsars, cyclotron lines provide a direct method of measuring the magnetic field strength at the site of the emission: $B_{12}=(1+$ $z) E_{\text {cycl }} / 11.6 \mathrm{keV}$, where $B_{12}$ is the magnetic field strength in units of $10^{12} \mathrm{G}, z$ is the gravitational redshift, and $E_{\text {cycl }}$ is the measured energy of the cyclotron line centroid. Her X-1 was the first neutron star for which the direct measurement of the strength of the Teragauss magnetic field was obtained. The line was originally observed at $\sim 42 \mathrm{keV}$ (Trümper et al. 1978). This gave an estimate of the field strength of $B \sim 3 \times 10^{12} \mathrm{G}$.

\section{Long-term behaviour of the cyclotron line energy}

Figure 1 shows the historical development of the cyclotron line energy $E_{\text {cycl }}$ (averaged over $1.24 \mathrm{~s}$ pulse phase) as it was measured with different instruments. The plot includes the data from the compilation in Staubert et al. (2007) plus our latest measurement of the line energy during the INTEGRAL observation in September 2007. Two features can be noticed in the plot: (1) an apparent jump in the line energy from $\sim 34$ to $\sim 42 \mathrm{keV}$, first pointed out by Gruber et al. (2001), appearing somewhere between 1991 and 1993, (2) an apparent decrease of the line energy with time after 1991.

At the moment there is no plausible explanation for the jump in the line energy. Staubert et al. (2007) have speculated that the abrupt jump might be associated with a possible transition 


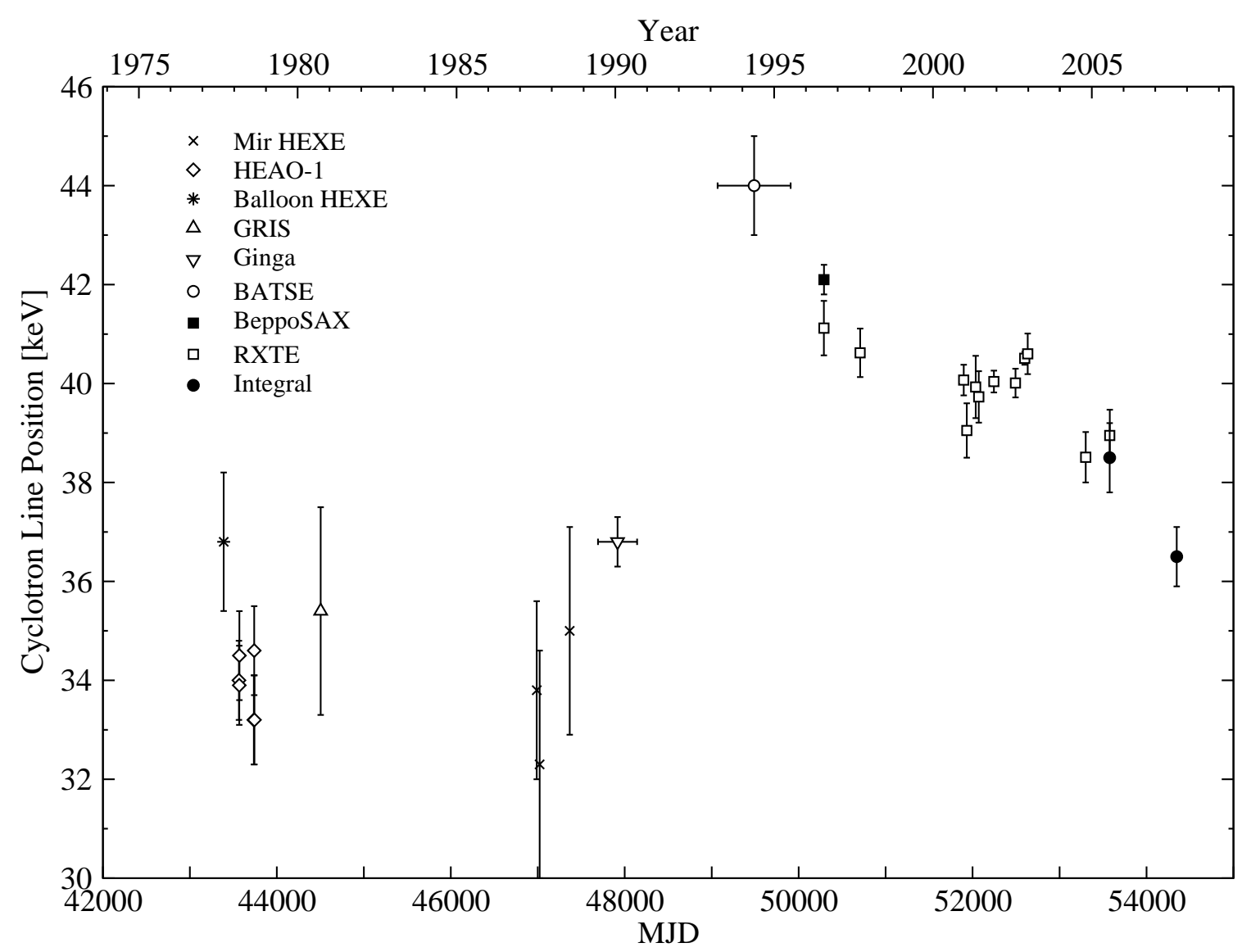

Figure 1: The centroid energy of the phase-averaged cyclotron line in Her X-1 since its discovery (see Staubert et al. 2007, and references therein). The rightmost data point corresponds to the latest INTEGRAL observation in September 2007.

of the pulsar from local super-Eddington to sub-Eddington accretion regime. Another possible explanation for the jump might be a sudden restructuring of the accretion mound at a polar cap of the neutron star (as a result of accumulated magnetic stresses in the accreted matter). The time scale for such events, however, seems to be rather long, thousands of years (see e.g. Brown \& Bildsten 1998; Litwin et al. 2001).

The apparent downward trend in the line energy after 1991 is discussed in Staubert et al. (2007). The authors show, however, that the trend is most likely an artifact introduced by the general variability of the source and a newly discovered positive correlation between the X-ray luminosity of the source and the cyclotron line energy $E_{\text {cycl }}$. The downward trend is then mostly due to the random way by which the data happened to be taken and the locally measured flux values, and not a secular decrease.

The newest INTEGRAL measurement of the line energy (the rightmost data pount in Fig. 1) which was not available to Staubert et al. 2007, however, seems to be again in line with a downward trend. Therefore, we would not rule out that a secular decrease of the line energy of some magnitude might be a separate phenomenon on top of the positive correlation between the line energy and $E_{\text {cycl. }}$. 


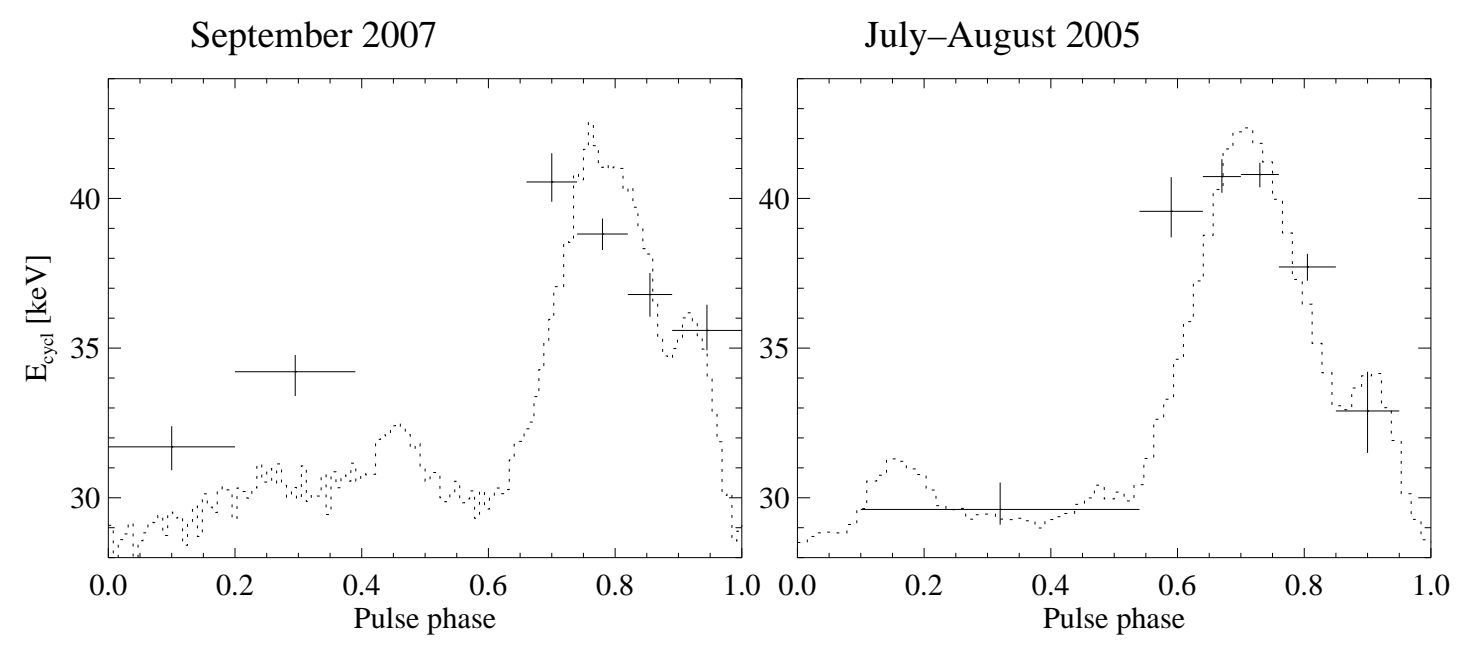

Figure 2: The centroid energy of the cyclotron line $E_{\text {cycl }}$ as a function of pulse phase measured with $I N$ TEGRAL in September 2007 (left) and July-August 2005 (right, Klochkov et al. 2008). The dashed lines represent the corresponding X-ray pulse profiles of the source in the energy range $20-100 \mathrm{keV}$.

\section{Pulse-phase behaviour of the cyclotron line energy}

It is known that the spectral parameters of Her X-1, including the energy of the cyclotron absorption line, vary with rotational phase of the neutron star (pulse phase) (see e.g. Voges et al. 1982; Soong et al. 1990a). The INTEGRAL instruments allowed us to study this variability with better statistics than it was possible before (the pulse-phase resolved analysis of the RXTE data is ongoing). Figure 2 represents the dependence of $E_{\mathrm{cycl}}$ on pulse phase which we measured with INTEGRAL in September 2007 (left) and in July-August 2005 (right).

The pulse-phase variability of $E_{\text {cycl }}$ during the latest INTEGRAL observations (in 2007) is similar to that observed with INTEGRAL in 2005 . The large amplitude of variation in $E_{\text {cycl }}$ $(\sim 20 \%)$ can be explained assuming a complicated structure of the emission pattern on the neutron star surface due to the presence of non-dipole components of the star's magnetic field (Klochkov et al. 2008, and references therein). The maximal energy of the line is similar in both observation $(\sim 41 \mathrm{keV})$ and is reached somewhere around the main peak of the pulse profile. The minimal value of $E_{\mathrm{cycl}}$ (reached in the interpulse) during the observation in 2007 is somwhat larger than during the observations in 2005. The pulse-phase averaged energy of the cyclotron line, however, is substantially higher during the observations in 2005 than in 2007 (see Fig. 1). The reason for this difference is the fact that the maximum of $E_{\text {cycl }}$ during the 2005 observations coincides with the maximum of the pulse profile which provides the largest contribution to the average X-ray spectrum. During the 2007 observations, however, the maximum of $E_{\mathrm{cycl}}$ appeares slightly before the peak in the pulse profile, whereas at the maximum of the profile the energy of the line is lower.

The described effect demonstrates that the pulse-phase averaged energy of the cyclotron line might significantly depend on the shape of the pulse profile (i.e. pulse phase of the main peak with respect to the maximum in $\left.E_{\text {cycl }}\right)$. This effect might be partially responsible for the observed variation of the pulse-averaged $E_{\text {cycl }}$ with time. Again, these variations may not be of secular nature. It is, therefore, important to continue the monitoring of Her X-1 with instruments which 
Table 1: Quadratic ephemeris of Her X-1 determined from a common fit of the historical observations and the new data from RXTE and INTEGRAL. The uncertainties in parentheses (68\%) refer to the last digit(s).

\begin{tabular}{ll}
\hline \hline$T_{\frac{\pi}{2}}(0)[\mathrm{MJD}(\mathrm{TDB})]$ & $46359.871944(6)$ \\
$P_{\text {orb }}[$ days $]$ & $1.700167589(2)$ \\
$\dot{P}_{\text {orb }}[\mathrm{d} / \mathrm{d}]$ & $-4.82(13) \times 10^{-11}$ \\
\hline
\end{tabular}

allow to perform pulse-phase resolved X-ray spectral studies, such as those onboard INTEGRAL, RXTE and Suzaku.

\section{Secular decrease of the orbital period}

We have updated the orbital ephemeris of Her X-1 by performing a timing analysis of all observations of sufficient quality done by RXTE and INTEGRAL during the last 12 years. The previous best ephemeris data were from Deeter et al. 1981 and Stelzer et al. 1997. Combining our results with previously published ones (e.g., Deeter et al. 1981, 1991; Wilson et al. 1994, and references therein), we were able to significantly improve the values for $T_{\frac{\pi}{2}}, P_{\text {orb }}$ and $\dot{P}_{\text {orb }}$. A full description will appear in Staubert et al. (2009b).

To calculate the new ephemeris we fitted the times of mean upper conjunction $T_{\frac{\pi}{2}}$ (the times when the mean orbital longitude of the neutron star is $90^{\circ}$ ) measured with RXTE and INTEGRAL and the historical values with the quadratic formula:

$$
T_{\frac{\pi}{2}}(n)=T_{\frac{\pi}{2}}(0)+n P_{\text {orb }}+\frac{1}{2} n^{2} P_{\text {orb }} \dot{P}_{\text {orb }},
$$

where $n$ is the orbital cycle number; $T_{\frac{\pi}{2}}(0), P_{\text {orb }}$, and $\dot{P}_{\text {orb }}$ are free fit parameters. The best-fit quadratic model is shown with the solid line in Fig. 3. The fit gives $\chi_{\text {red }}^{2}=2.1$ for 27 d.o.f.

As an alternative to the solution with continuous decrease of the orbital period, Deeter et al. (1981) proposed a solution assuming a constant $P_{\text {orb }}$ with a sudden jump around 1983. It was motivated by the unusually long Anomalous Low State (ALS) of the source that took place in 1983-1984. Deeter et al. (1981) suggested that if the change in the orbital period is confined to the ALS then both phenomena can be explained by a sudden temporary increase in the rate of mass transfer from the normal companion to the neutron star. Following this suggestion, we performed a separate fit of the data before and after 1983 with a linear ephemeris (the dashed lines in Figure 3). The fit gives $\chi_{\text {red }}^{2}=4.3$ for 27 d.o.f. which is more than two times larger than that for the quadratic ephemeris. We can, therefore, reject the sudden change of the orbital period in 1983. We also note that from our disk-stream coupling model (Shakura et al. 1999; Klochkov et al. 2006) we expect a decrease of the mass transfer to the neutron star during ALSs rather than an increase.

The best-fit values of $\dot{P}_{\text {orb }}, T_{\frac{\pi}{2}}(0)$, and $P_{\text {orb }}$ comprise a new orbital ephemeris of Her X-1 that can be used to calculate the orbital period $P_{\text {orb }}$ and the time $T_{\frac{\pi}{2}}$ for any particular observation. Their values are listed in Table 1 


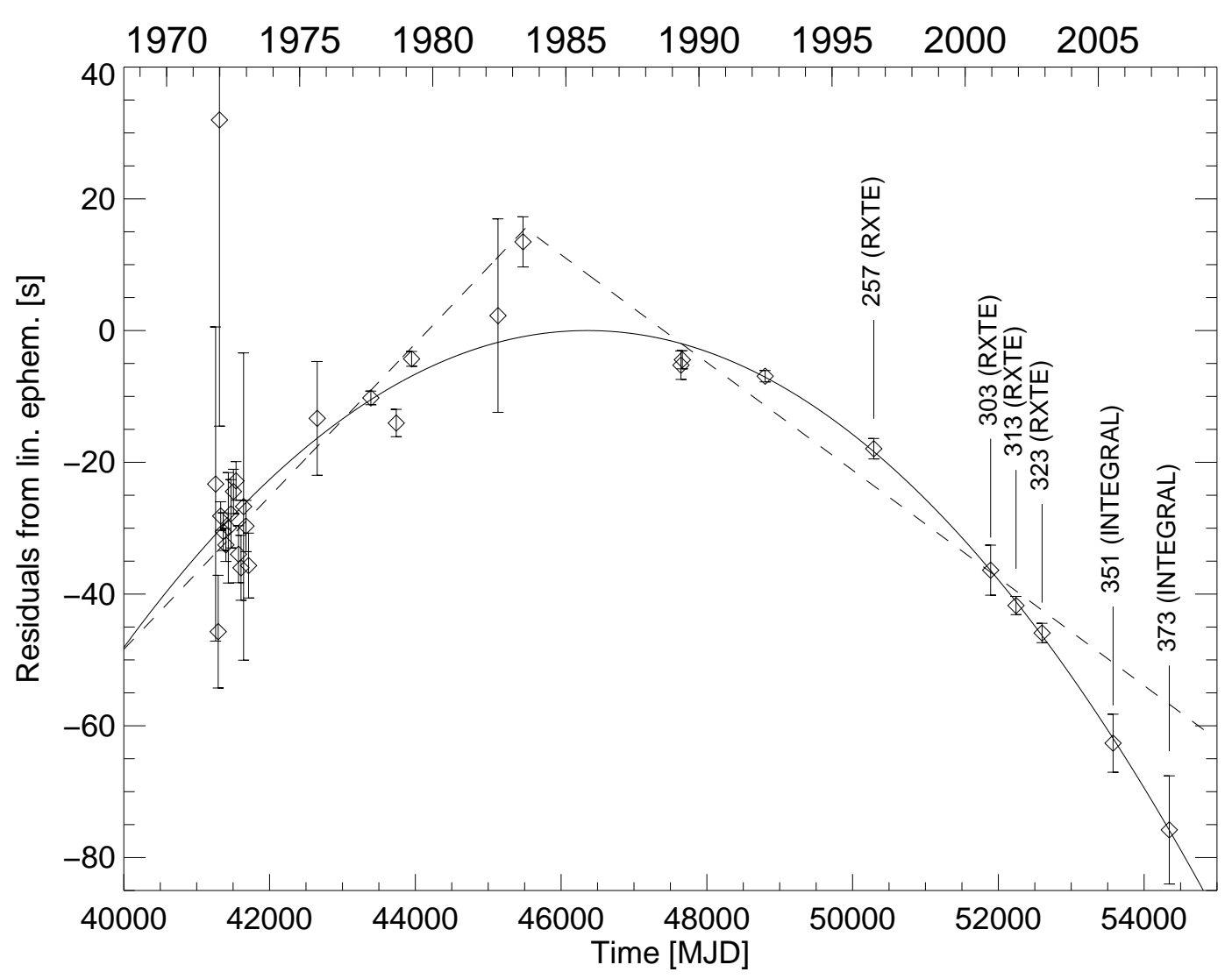

Figure 3: Residuals of $T_{\frac{\pi}{2}}$ after subtracting the linear part of the quadratic best-fit ephemeris (Klochkov 2007; Staubert et al. 2009b). For RXTE observations analyzed in this work 35 d cycle numbers are indicated (according to the $35 \mathrm{~d}$ cycle counting convention in Staubert et al. 1983). The dashed line shows a fit by the linear ephemeris with a sudden jump of the orbital period around 1983.

\section{Summary and conclusions}

Her X-1 is the only highly magnetized accreting pulsar for which repeated observations over long period of time exist. This allowed us to observe the variation in the energy of the cyclotron line (one of the most interesting features of accreting pulsars) on a long (20 yr) timescale. The RXTE and INTEGRAL data have revealed a positive correlation between the X-ray flux of the source and the pulse-phase averaged cyclotron line energy $E_{\text {cycl }}$ (Staubert et al. 2007). The apparent decrease of this energy with time, which is emphasized by the new INTEGRAL measurement of 2007 (rightmost point in Fig. 1), may indicate that there is indeed a contribution from a true secular decrease after 1991 (in addition to the artifact related to the large variations in flux and the correlation with $\left.E_{\mathrm{cycl}}\right)$. Such a secular decrease might be an indication of a slowly changing structure of the accretion mound due to the constant accumulation of matter accreted from the companion. A third factor is the variability of the pulse profiles and the strong modulation of the cyclotron line energy with pulse phase, the systematics of which are not yet understood. We stress that it is particularly important to continue the X-ray monitoring of the source in order to separate the various effects and to determine their relative significance. 
Our study of the pulse-phase dependence of the cyclotron line energy during the INTEGRAL observations in 2005 and 2007 confirmed that the amplitude of variation in $E_{\text {cycl }}$ with the rotational phase of the pulsar is very large, up to $\sim 20 \%$. As it was shown in Sect. 3, the pulse-averaged value of the line energy in this case can be significantly affected by changes in the shape of the pulse profile. Therefore, variations in pulse profile can be (at least partially) responsible for the observed variability of the pulse-averaged energy of the cyclotron line. We plan to perform a coherent pulsephase resolved analysis of all RXTE data available to us in order to explore this issue.

The RXTE and INTEGRAL monitoring of the source allowed us to perform a joint timing analysis of all available data including the historical observations of Her X-1 with previous satellites (Staubert et al. 2009b). As a result, we confirm the continuous decay of the system's orbit and rejected a sudden change of the period during the Anomalous Low State in 1983. The orbital ephemeris of Her X-1 (including the value of $\dot{P}_{\text {orb }}$ ) were substantially improved (see Table 1) with respect to the previous ephemeris provided by Deeter et al. (1981) and Stelzer et al. (1997).

We acknowledge the support through DFG grants Sta 173/31 and 436 RUS 113/717/0-1 and the corresponding RBFR grants RFFI-NNIO-03-02-04003 and RFFI 06-02-16025, as well as DLR grant 50 OR 0302. We thank L. Rodina for her contributons to the data reduction and analysis.

\section{References}

Brown, E. F. \& Bildsten, L. 1998, ApJ, 496, 915

Crampton, D. 1974, ApJ, 187, 345

Deeter, J. E., Boynton, P. E., Miyamoto, S., et al. 1991, ApJ, 383, 324

Deeter, J. E., Pravdo, S. H., \& Boynton, P. E. 1981, ApJ, 247, 1003

Deeter, J. E., Scott, D. M., Boynton, P. E., et al. 1998, ApJ, 502, 802

Gerend, D. \& Boynton, P. E. 1976, ApJ, 209, 562

Giacconi, R., Gursky, H., Kellogg, E., et al. 1973, ApJ, 184, 227

Gruber, D. E., Heindl, W. A., Rothschild, R. E., et al. 2001, ApJ, 562, 499

Howarth, I. D. \& Wilson, B. 1983, MNRAS, 202, 347

Klochkov, D. 2007, PhD thesis, University of Tübingen, Germany, http://w210.ub.unituebingen.de/volltexte/2007/3181/pdf/disser.pdf

Klochkov, D., Staubert, R., Postnov, K., et al. 2008, A\&A, 482, 907

Klochkov, D. K., Shakura, N. I., Postnov, K. A., et al. 2006, Astronomy Letters, 32, 804

Liller, W. 1972, IAU Circ., 2415, 1

Litwin, C., Brown, E. F., \& Rosner, R. 2001, ApJ, 553, 788 
Scott, D. M., Leahy, D. A., \& Wilson, R. B. 2000, ApJ, 539, 392

Shakura, N. I., Prokhorov, M. E., Postnov, K. A., \& Ketsaris, N. A. 1999, A\%A, 348, 917

Soong, Y., Gruber, D. E., Peterson, L. E., \& Rothschild, R. E. 1990a, ApJ, 348, 641

Soong, Y., Gruber, D. E., Peterson, L. E., \& Rothschild, R. E. 1990b, ApJ, 348, 634

Staubert, R., Bezler, M., \& Kendziorra, E. 1983, A\&A, 117, 215

Staubert, R., Klochkov, D., Postnov, K., et al. 2009a, A\&A, 494, 1025

Staubert, R., Klochkov, D., \& Wilms, J. 2009b, A\&A, [accepted]

Staubert, R., Shakura, N. I., Postnov, K., et al. 2007, A\&A, 465, L25

Stelzer, B., Staubert, R., Wilms, J., et al. 1997, in American Institute of Physics Conference Series, Vol. 410, Proceedings of the Fourth Compton Symposium, ed. C. D. Dermer, M. S. Strickman, \& J. D. Kurfess, 753

Tananbaum, H., Gursky, H., Kellogg, E. M., et al. 1972, ApJ, 174, L143

Trümper, J., Kahabka, P., Oegelman, H., Pietsch, W., \& Voges, W. 1986, ApJ, 300, L63

Trümper, J., Pietsch, W., Reppin, C., et al. 1978, ApJ, 219, L105

Voges, W., Pietsch, W., Reppin, C., et al. 1982, ApJ, 263, 803

Wilson, R. B., Finger, M. H., Pendleton, G. N., Briggs, M., \& Bildsten, L. 1994, in American Institute of Physics Conference Series, Vol. 308, The Evolution of X-ray Binariese, ed. S. Holt \& C. S. Day, 475 